\title{
Research Letter \\ Correlation between Compressive Strength and Rheological Parameters of High-Performance Concrete
}

\author{
Aminul Islam Laskar ${ }^{1}$ and Sudip Talukdar ${ }^{2}$ \\ ${ }^{1}$ Civil Engineering Department, National Institute of Technology, Silchar 788010, India \\ ${ }^{2}$ Civil Engineering Department, Indian Institute of Technology, Guwahati 781039, India
}

Correspondence should be addressed to Sudip Talukdar, staluk@iitg.ernet.in

Received 23 July 2007; Accepted 25 October 2007

Recommended by Luigi Nicolais

Compressive strength is greatly influenced by the performance of concrete in its fresh stage such as uniform mixing, proper compaction, resistance to segregation during transporting and placing. Attempt has, therefore, been made to correlate compressive strength to the rheological behavior of high performance concrete with a modified setup of parallel plate rheometer. Modified setup considers the shearing of concrete at the centre of the cylindrical container that takes into account the resistance between concrete and the vertical side of the wall. It has been observed that compressive strength increases steeply as the yield strength increases up to a certain level. Plastic viscosity, however, shows optimum value for maximum compressive strength.

Copyright (C) 2007 A. I. Laskar and S. Talukdar. This is an open access article distributed under the Creative Commons Attribution License, which permits unrestricted use, distribution, and reproduction in any medium, provided the original work is properly cited.

\section{INTRODUCTION}

High performance concretes (HPC) are concretes with properties or attributes that satisfy the performance criteria. HPC requires dense, void-free mass with full contact with reinforcing bars. Workability has to be compatible with these fundamental needs to achieve high performance. To do so, mix should be such easy to vibrate and fluid enough to pass through congested reinforcement. HPC possesses three characteristics: high strength, high durability, and high workability. A minimum slump of $100 \mathrm{~mm}$ is therefore preferred [1]. Durability is related to low permeability. High strength and low permeability, although not necessarily concomitant, are linked to one another because high strength requires low volume of pores. Thus, remaining two characteristics that need careful control and monitoring at the production stage are high strength and high workability. Workability of fresh HPC is characterized by its rheological parameters, namely, yield stress and plastic viscosity. In measuring rheological properties of concrete, it is essential to know the flow properties over a wide range of shear stresses and shear strain rates. The most commonly used model used in concrete is the Bingham model [2] where the flow is described by

$$
\tau=\tau_{o}+\mu \dot{\nu},
$$

where $\tau_{o}=$ yield stress, $\mu=$ plastic viscosity, and $\dot{v}=$ shear strain rate. Yield stress and plastic viscosity are considered to be fundamental parameters of fresh concrete rheology. Rheological parameters vary depending on the measurement techniques used [3]. Parallel plate rheometer is claimed to be unique because it allows calculation of rheological parameters in fundamental units of stress and strain rate [4]. In parallel plate rheometer like BTRHEOM, it is assumed that vertical wall of cylindrical container does not perturb the motion of concrete [3]. In reality, a friction takes place between concrete and the vertical wall though the friction may be low compared to the yield stress in concrete. The present study considers a modified setup of parallel plate geometry where concrete is made to shear at the middle of the cylinder. Thus, frictional resistance between wall and concrete which were earlier neglected has been taken into account to develop the expression for torque.

Compressive strength (cube or cylinder) of concrete is one of the most important hardened properties. Obviously, one might expect that there is a good correlation between rheological parameters and the compressive strength of concrete. Review of literature reveals that little work has been done so far in this area. Chidiac et al. [5] reported that it is possible to correlate rheological properties of fresh concrete to its hardened properties such as core strength, ultrasonic 
pulse velocity, pull-off strength, air permeability. and sorptivity. However, the concrete investigated in the work in [5] was usual or normal strength concrete (target strength was $20 \mathrm{MPa}$ ) with high water-cement ratio ranging between $0.65-0.80$. It is worthwhile to mention in this connection that high strength-high performance concrete has water-binder ratio normally less than 0.45 and compressive strength falling in the range of 42-100 $\mathrm{MPa}$ [1]. Therefore, attempt has been made to correlate compressive strength of high strength-high performance concrete and its fresh rheological properties.

\section{EXPERIMENTAL PROGRAM}

\subsection{Materials}

Two different categories of soft high performance mixtures were used in the present study: one with Polycarboxylic Ether Polymer (PC) as HRWRA and the other with Sulfonated Naphthalene Polymer (SN) with set retarding effect. The cement used throughout the experiment was Ordinary Portland Cement (OPC). The 28-day compressive strength and specific gravity of cement were $50.2 \mathrm{~N} / \mathrm{mm}^{2}$ and 3.10 , respectively. Locally available alluvial sand (medium; specific gravity $=2.6$ ) from a same pit was used. Sand stockpile was stored inside the laboratory throughout the experimental investigation. Sand was not sieved and was used as received directly from the stockpile unless otherwise mentioned. Crushed stone aggregate (specific gravity $=2.6$ ) of nominal maximum size $16 \mathrm{~mm}$ was collected and stored in the laboratory. Aggregates were not sieved and were used as received directly from the stockpile. Ordinary tap water was used for all the mixes to prepare fresh concrete. The batch size was 16 liters in all the cases.

\subsection{Experimental setup}

The rheological measurements were performed with the modified setup of parallel plate rheometer. The schematic diagram is shown in Figure 1. It consists of a 150-mm-diameter flat circular vane plate driven by an AC single-phase induction motor through a gearbox. Vane plate is mounted coaxially with a cylindrical container (effective diameter = $270 \mathrm{~mm}$ ) with sleeve and bearing arrangement to ensure accurate alignment. The cylindrical container is provided with vertical ribs of $20 \mathrm{~mm}$ projection at a pitch of $60 \mathrm{~mm}$ along the circumference. Ribs are also welded at the bottom of the cylinder. The effective gap between the bottom and the shearing surface is $75 \mathrm{~mm}$. The effective concrete height above the vane plate is also $75 \mathrm{~mm}$. The no-slip condition at top of the cylinder is achieved by providing 20-mm-high mesh of blades. The blade mesh can be detached as and when necessary to facilitate convenience in cleaning. The torque of the motor and hence the vane plate is controlled by varying input voltage with a 10 -ampere $\mathrm{AC}$ variac. The number of revolution of the vane plate is measured automatically with a noncontact infrared digital tachometer, by focusing at the retroreflective tape glued to the spindle. The spindle has a pulley welded to it that is used for calibration purpose only. The torque provided by the rheometer was calibrated

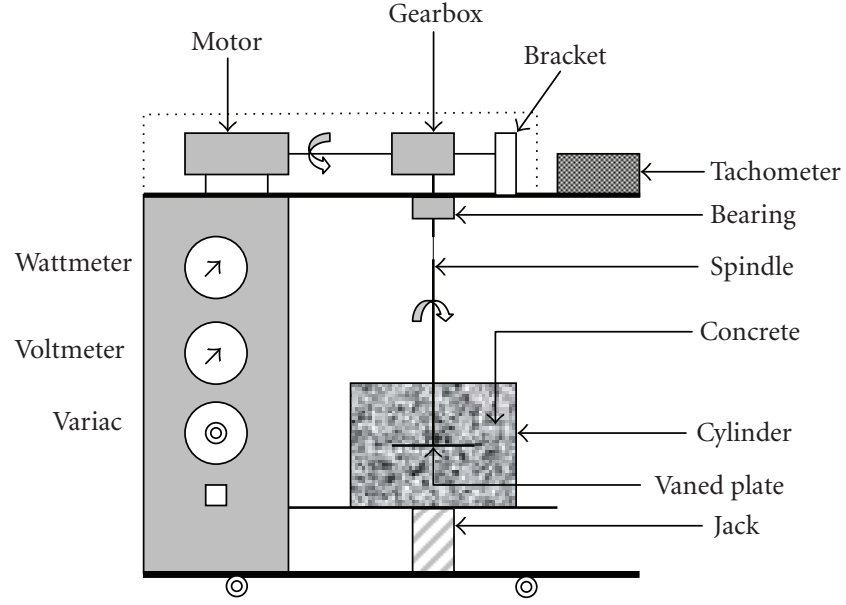

FIgURE 1: Schematic diagram of the present set up.

in terms of input AC voltage. A spring balance anchored to a fixed object is fitted to the pulley of the spindle. When the motor is switched on, the spring balance blocks its rotor and the spring balance reading is noted. This arrangement gives the braking torques at different voltages. The rheometer has been validated by testing Magneto-rheological (MRF 132DG) fluid by HAAKE RS1 rheometer and compared with the results obtained using the present rheometer. In the present study, stepwise increasing shear stress sequence followed by a decreasing shear stress was used and the down curve was taken to draw the flow curve. Concrete was sheared at each step for 30 seconds and readings were taken at the end of each period. Care was taken during the measurements so that shear strain rate did not exceed $10 \mathrm{sec}^{-1}$ in any case $[6,7]$. Otherwise, particle migration of unknown magnitude may affect the test results. Plug flow, if any, was corrected graphically. Any flow curve giving negative value of yield stress and $R^{2}$ value less than 0.98 was rejected and repeated with a new batch.

\subsection{Mixing}

Concrete was mixed in a tilting mixer (laboratory type). The weight of the materials was taken with a digital weighing balance. Mixing sequence was as follows.

(i) Mix coarse aggregate, fine aggregate cement for two minutes.

(ii) Add water during mixing and mix for two minutes more.

(iii) Stop mixing for one minute.

(iv) Add HRWRA to the mix and mix for three minutes.

(v) Pour the concrete mix.

\subsection{Testing procedure}

The prepared concrete was transferred to the cylinder with a trowel from the same height every time. The rheological test was carried out exactly after 15 minutes from the instant of addition of water. After one observation, the mix was 


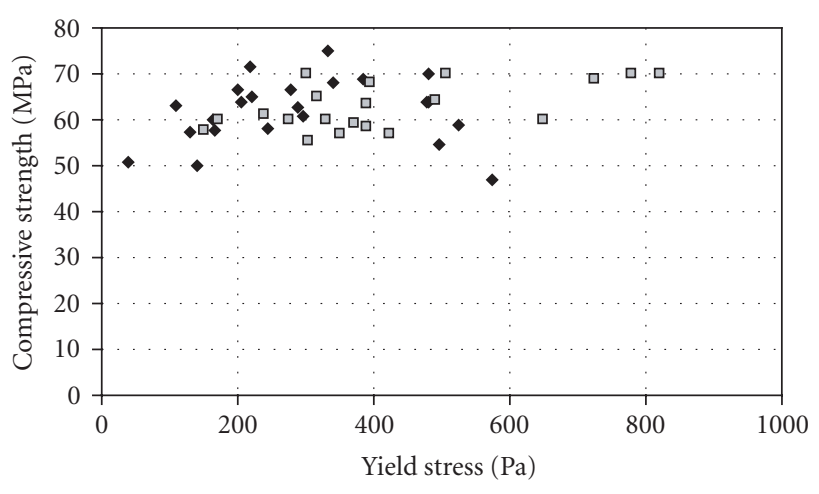

- Mix with PC

- Mix with SN

FIGURE 2: Variation of compressive strength with yield stress.

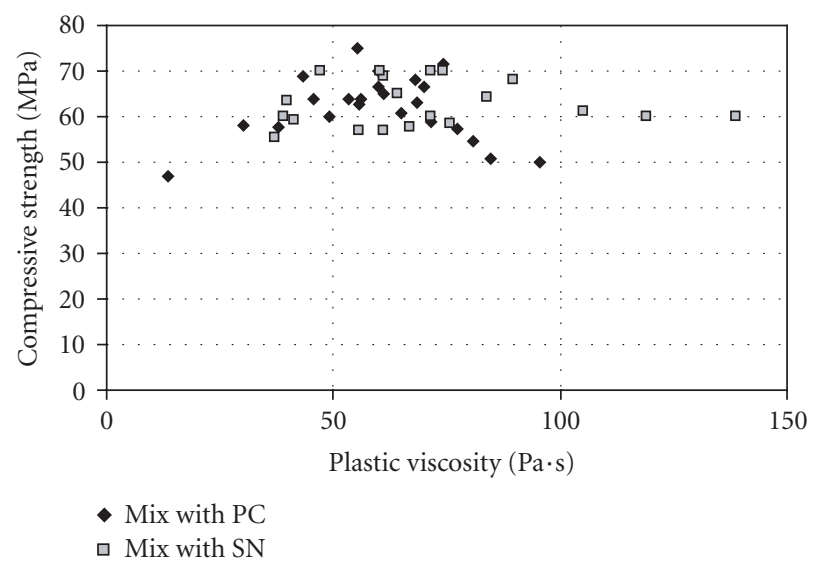

FIgURE 3: Variation of compressive strength with plastic viscosity.

rejected. Each time, a new batch of concrete with the desired composition for a particular mix was prepared. The mixing sequence and the time at which the rheological test was performed were identical for each batch and for all mixes.

\section{RESULTS AND DISCUSSION}

Figures 2 and 3 show the variation of compressive strength with respect to yield strength and plastic viscosity, respectively. Compressive strength in the present case is the average of 28-day compressive strength of three $15-\mathrm{cm}$ cubes cured under water. It may be observed that initially compressive strength increases steeply as yield stress increases. Further increase in yield stress beyond certain point reduces compressive strength in case of mix with PC. In mixtures containing SN as HRWRA, compressive strength also increases steeply up to yield stress equal to approximately $500 \mathrm{~Pa}$ and then remains unchanged with the increase in yield stress. It may also be observed that optimum values of plastic viscosity exist for both the categories of mixes. Compressive strength, however, decreases at a slower rate in mix with SN beyond optimum value.

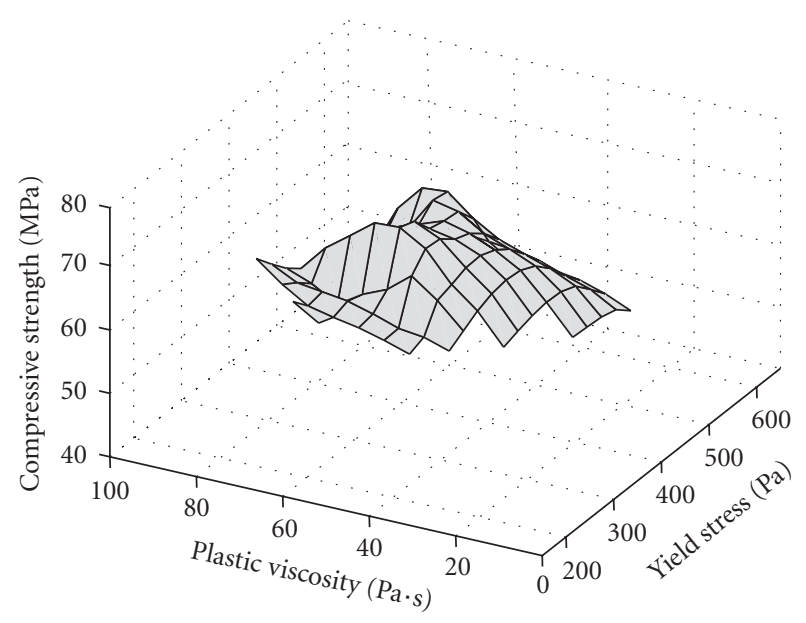

FIGURE 4: Variation of compressive strength with yield stress and plastic viscosity with PC.

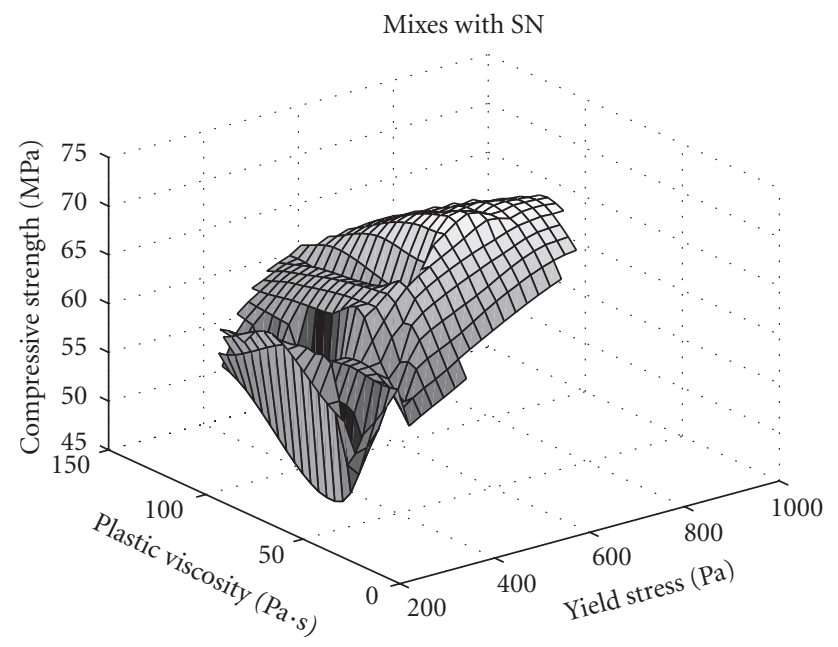

FIGURE 5: Variation of compressive strength with yield stress and plastic viscosity with $\mathrm{SN}$.

In fact, complete rheological property of concrete is described by combination of yield stress and plastic viscosity simultaneously. The influence of mixture proportions and properties of ingredients are all contained in yield stress and plastic viscosity. Hence, for better representation, compressive strength is plotted against yield stress and plastic viscosity as 3D surface (see Figures 4 and 5). Smooth surfaces could have been obtained with large number of data. For plastic viscosity between $50-75 \mathrm{~Pa} \cdot \mathrm{s}$, and yield stress between 300 $400 \mathrm{~Pa}$, compressive strength is the highest in mix with PC, whereas in mixtures with $\mathrm{SN}$, compressive strength shows highest value when plastic viscosity lies between 60-90 Pa.s and yield stress is greater than $500 \mathrm{~Pa}$. It is worth to be mentioned here that concrete in these range of rheological parameters are very much workable. 


\section{CONCLUSION}

A modified setup for parallel plate has been used to carry out the experimental investigation on high performance concrete. In the present setup, concrete is sheared from the centre instead of shearing from top or bottom of the material. Resistance offered by the vertical wall of the container has also been taken into account. Rheological parameters and compressive strength of two categories of high performance concrete were determined. The ranges of Bingham parameters and compressive strength studied in the present investigation are as follows:

(i) Yield stress: 40-820 Pa;

(ii) Plastic viscosity: $15-140 \mathrm{~Pa} \cdot \mathrm{s}$; and

(iii) Compressive strength (28 day): 45-78 MPa.

The following conclusions may be derived from the present work.

(a) In mixtures containing SN as HRWRA, compressive strength increases steeply up to yield stress equal to approximately $500 \mathrm{~Pa}$ and then remains unchanged with the increase in yield stress. Compressive strength shows peak value when plastic viscosity lies between 60-90 Pa.s.

(b) For plastic viscosity between $50-75 \mathrm{~Pa} \cdot \mathrm{s}$, and yield stress between $300-400 \mathrm{~Pa}$, compressive strength shows peak value in mixtures with PC. Further increase in yield stress beyond peak value reduces compressive strength.

(c) Optimum values of plastic viscosity exist for both the categories of mixes for maximum compressive strength.

\section{REFERENCES}

[1] E. G. Nawy, Fundamentals of High Performance Concrete, John Wiley \& Sons, New York, NY, USA, 2nd edition, 2001.

[2] G. H. Tattersall, Workability and Quality Control of Concrete, E \& FN Spon, London, UK, 1991.

[3] C. F. Ferraris and L. E. Brower, "Comparison of concrete rheometers: international tests at MB," Tech. Rep. NISTIR 7154, National Institute of Standards and Technology, Cleveland, Ohio, USA, 2004.

[4] V. S. Ramachandran and J. J. Beaudoin, Handbook of Analytical Techniques in Concrete Science and Technology, Williams Andrew, Norwich, NY, USA, Indian edition, 2006.

[5] S. E. Chidiac, O. Maadani, A. G. Razaqpur, and N. P. Mailvaganam, "Correlation of rheological properties to durability and strength of hardened concrete," Journal of Materials in Civil Engineering, vol. 15, no. 4, pp. 391-399, 2003.

[6] R. S. Szecsy, "Concrete rheology," Ph.D. dissertation, University of Illinois at Urbana-Champaign, Urbana, Ill, USA, 1997.

[7] N. Roussel, "A thixotropy model for fresh fluid concretes: theory, validation and applications," Cement and Concrete Research, vol. 36, no. 10, pp. 1797-1806, 2006. 

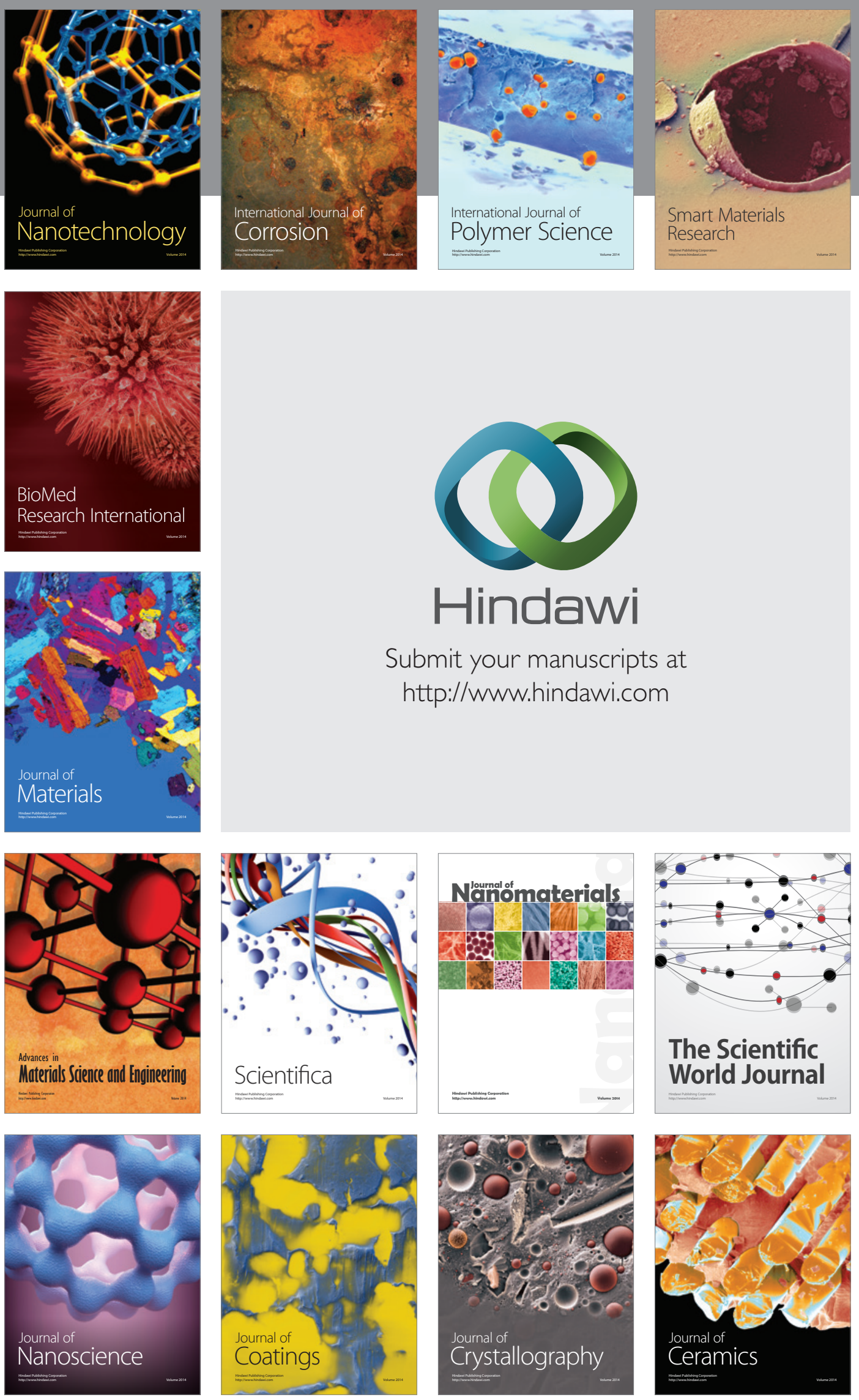

The Scientific World Journal

Submit your manuscripts at

http://www.hindawi.com

\section{World Journal}

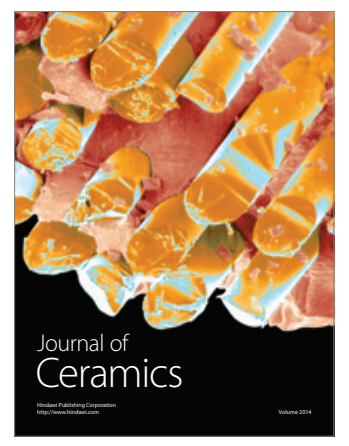

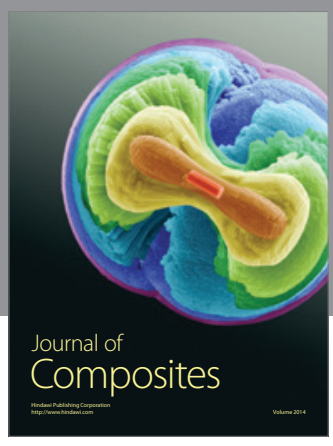
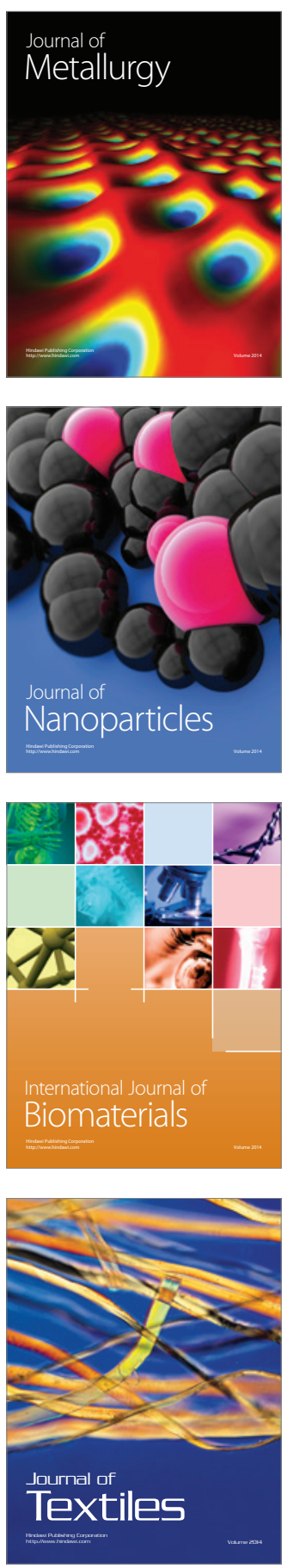